\title{
HUMAN-ROBOT COLLABORATION: TECHNICAL ISSUES FROM A VIEWPOINT OF HUMAN-CENTERED AUTOMATION
}

\author{
Tetsuo Sawaragi and Yukio Horiguchi \\ Department of mechanical Engineering and Science, Graduate School of Engineering \\ Kyoto University \\ Yoshida Honmachi, Sakyo, Kyoto 606-8502, Japan. \\ \{sawaragi, horiguchi\}@me.kyoto-u.ac.jp
}

\begin{abstract}
In this article, after surveying the problems incurred by the conventional technology-centered automation in a variety of fields, we put an emphasis on the fact that a concept of "sociality" is really needed to form the ideal human-automation and/or human-robot relations emerging out of active interactions. Then, some key technical issues are to be presented for realizing such socially-centered automation and establishing the human-automation and/or human-robot collaboration. Brief introductions of the ongoing works by the author's group are also presented.
\end{abstract}

Keywords: Human-centered automation, human-robot collaboration, sociality, ecological interface design.

\section{INTRODUCTION}

In contrast to the conventional technology-centered design of artifacts (e.g., automation systems), now we should consider ideal human-computer relationships with respect to their "interactions". This means that the human and technological subsystems like automations should be structured and designed to work in mutually cooperating ways guaranteeing a user's usability. For this purpose, progressive system redesigns are needed with respect to human machine interactions architecting to increase system reliability and transparency by allowing a human user's proactive participation, rather than by eliminating the human out of the loop.

In a field of manufacturing, an automation tool has contributed much with respect to design and production processes. However, this trend has been drastically shifted currently; a shift from a large-scaled, expensive full automation systems towards a more compact, economical, semi-autonomous and reconfigurable devices (i.e., Low Cost Automation). This shift has been caused by the ongoing change of the needs of high-mix low-volume production in contrast to the conventional mass production. As a result of this, a new style of human-automation collaboration should be established.

When the robots were introduced in factories, there existed difference in the approaches between Japan and Europe and the United States. In Japan human workers took a role of arranging, controlling and setting up the processes of production so that the robots could work in continuation. On the other hand, in Europe and the United States robots were regarded as a tool for alternating and replacing human workload by machines, and human workers did not change for the robots, which resulted in the generation of many "islands of automation". In this sense, we can find the origin of human-automation collaboration in Japanese factories.

Now the human-automation collaboration is really needed because of the following reasons. At first the manufacturing process should be flexible and reconfigurable admitting human participation. Secondly, by letting a human remain in the control loop, human "deskilling" problem will be solved. Thirdly, automation should work so that it could enhance the human capability as well as human motivation. The validity of the above human-centered manufacturing has been actually verified by the success of the cell production systems in Japanese factories.

In the design of human-automation systems, cognitive behaviors and strong affective elements come into play through the human user/operator and serious deficiencies can become apparent after system is delivered and is put to work. In this article, after surveying the problems incurred by the conventional technology-centered automation in a variety of fields, we put an emphasis on the fact that a concept of "sociality" is really needed for truly establishing human-centered automation [1, 2]. Then, some key technical issues are presented for realizing such socially-centered automation and establishing the human-automation and/or human-robot collaboration. Finally, brief introductions of the ongoing works by the author's group are also presented.

\section{TECHNOLOGY ENABLING USERS' PARTICIPATION}

The conventional design principle of human-machine systems has been based on the conception that a human user is a likely source of significant variance in system performance, then it is better to control this source of variance early and/or to minimize such human factors in system development. However, a human itself is too complex to be controlled, and the human is at the same time a source of creativity that sometimes exceeds out of the designer's restricted views. Thus, the system should be designed so that it can be possible both for a human and an automated agent to share knowledge about the other's 
operations and functioning, intent, and plans i.e., "mutual knowledge of intent".

It is widely accepted that extensive and proactive user participation is a key to success in both innovation promotion and the integration of human factors. Moreover, as a true partner of the user an automated system also has to have an analogous capability of "sociality" to be embedded within the interactions with the human user and to form a creative interactive domain together with him. In this sense, human and automated system should be looked as two parties coming from the same positions and having different expertise, and they must be regarded as equivalent partners, rather than one of them is regarded as superior to the other.

\section{EMERGING TECHNICAL ISSUES FOR HUMAN- AUTOMATION COORDINATION}

\subsection{Robotics Domain}

Currently robots are getting to work not only in the factory but also in our everyday life. Such robots are called social robots and they require more difficult technical problems to be solved as compared with the factory robots. The major difficulty is that their environments are not static but dynamically changing, so they have to be able to adapt to them. That is, the sources of the variable environment come from how the human users interact with them; users' preferences and their skill levels may change and develop, thus robots assisting them must be adaptable to them.

Due to the existence of the intentional others (i.e., human users), any robots cannot completely control the results that are brought about through the interactions with the human users. Thus, it is not realistic to pre-program all the possible behaviors of the robots by the designers, but the robots must be able to mimic the behavior of the others which it tracks by looking into others, and they should be able to form behaviors by themselves based on the accumulation of experiences. This is a "socialization" process in sociology: By interacting with the others and reflecting on this experience, robots have to reconstruct a definition of the reality, which causes them behave differently and changes the interaction patterns. This is iterated indefinitely, so the robots should be able to continue changing and keep variability. This is a social learning model for robots regarded as a continuous selfproduction process as internally-inspired activities.

\subsection{Automotive Domain}

Typical breakdowns of human-automation coordination are seen in aviation automation. Actually, there occur human errors induced by the difficulties in interactions between flight crews and cockpit automation. This kind of difficulty of intention-sharing between automation and human user is becoming serious also in the advanced automotive domain. Wherein, an interface problem is becoming of importance, but the consistency of the interface design with a user's mental model activated during the supervision of the dynamical systems is not verified from any formal perspectives.

The control logics embedded within an automated/ semi-automated machine are becoming too complex for a human user to correctly grasp their working status. That is, operation modes of the machine may change without notice, when a human user has to carefully pay attention to those mode-changes, which may incur extra mental workload. A typical example of this is a control logic embedded in ACC (Adaptive Cruise Control) system of the commercial automotive. Designers embed those logics within the machine from a designer's view (i.e., a machine model), but this is sometimes different from a user's view (i.e., a user model). The former is determined by the logic embedded in the machine system including automated functions that may sometimes be invisible and distal entities for a human user, while the latter is determined by action commands and display items of the interface system that are proximately available (i.e., directly perceivable and executable) to a user.

A user model is a possible abstraction of the machine model. A human driver cannot grasp all the details of the machine model specifications. Rather, he/she only highlights some of them, and what is highlighted is restricted to the ones recognizable through the available interface. Indeed such an abstraction may be possible in many ways, but a driver's user model determined by the interface must be correct and succinct abstraction of a machine model. If an incorrect and wrong abstraction occurs, it may be misguiding for a user's supervision of the automated machine's functioning status. Therefore, what is important here is how to evaluate a user model determined by a given interface with respect to whether that is a correct and succinct abstraction of the machine state or not. Composite model analysis can contribute to this evaluation as mentioned later.

\subsection{Designing and Manufacturing Domain}

Advanced CAD tools should have a capability of supporting a designer's creative task in a coherent way with the human expert designer does, and it has to enable not only design data but also design knowledge to be transferred among the design participants. Wherein, designers must be able to understand the intention of the original design precedents to successfully use them, but current design automation tools are insufficient for supporting such a task since they do not contain any design intention, decisional process information nor design knowledge. Design modification with lack of such expertise and with mis-understanding of the design precedents would lead to the design causing a malfunction of a product, which incurs a lot of time and expense cost.

The typical catastrophe caused by this was the JCO nuclear fuel production company accident happened in 1999, Japan. This happens because originally designed facilities were replaced with other available equipments to increase the efficiency of the work. Those alternative equipments were indeed functionally equivalent, but the safety design constraints implied in the original ones were 
not understood nor shared by the workers enough, which leaded to the accident. Thus, it is strongly requested to support workers to form an appropriate mental model and to perceive the system state through making visible the system state in terms of the safety boundaries as well as the system functional structure.

In Japanese companies such deep knowledge of design expertise have been transferred from expert designers to novice designers on the job training (OJT) directly through a man-to-man communication. However, current severe economic recession causes frequent re-assignment of expert designers to different work sections, which makes the transfer of knowledge/skills quite difficult. We are now seeking for alternative styles of knowledge transfer such as schooling education and formal documentation of design expertise, both of which are not going well as expected. The main reason why the experts are unwilling to transfer their knowledge is very simple; it is impossible to teach or formally describe design expertise by isolating that from the practice of design activity. In order to offer an effective support to a new design problem, design precedents must be stored together with implicit problem solving know-how used and realized in them.

\section{SOLUTIONS PROPOSED BY OUR GROUP}

\subsection{Social Reinforcement Learning}

Social learning is a process by means of which agents' acquisition of new information is caused or favored by their being exposed to one another in a common environment [3]. Socially intelligent agents are autonomous problem solvers that have to achieve their objectives by interacting with their environment and/or other conspecific autonomous entities including other artificial agents or natural humans. The interaction among these social agents is not only a means for information transferring, but also a strategy by which an agent learns from other intelligent entities.

In social learning, imitation learning is one of most important and common approaches that are adopted by human and other mammals. This imitation learning has been well-discussed by computer scientists as well as psychologists; the main topic wherein is to regard imitation learning accomplished by being merged with another machine learning technique of reinforcement learning (i.e., learning by watching others then by doing by themselves). Reinforcement learning (RL) is a learning algorithms that agents take an action in a certain situation so as to maximize a numerical reward signal. In RL, actions taken by an agent are followed by rewards or punishments from an environment. According to these rewards and punishments, this agent updates his knowledge about the corresponding states so that he can select the correct actions when it encounters the same state again. Moreover, actions of an agent may affect not only on the immediate reward, but also on the next situation and, through that, all subsequent rewards [4]. The final target of reinforcement learning algorithm is to establish a policy that can be applied to all possible states an agent may encounter.

Multiagent reinforcement learning (MRL) is a further extended learning framework in which each agent is an entity doing individual reinforcement learning and acquires knowledge through communication and interaction with its environment as well as with other learning agents who are living in the same environment. Social Reinforcement Learning (SRL) is a novel idea of machine learning that attempts to apply social learning findings from sociology to multiagent reinforcement learning. According to Maja J Mataric's viewpoint, social reinforcement learning includes three types: individual reinforcement, observing reinforcement and vicarious reinforcement. The details of those are shown in [5].

In order to verify the usefulness of SRL, we established a multiagent agent collaboration system composed of four software agents and a graphics simulation environment. The graphics server is a workstation located in the network, which simulates a ball's movement of rolling on a panel according to real world physical law. Individual agents are independent software programs distributed in the different computers in the same network and their task is to keep balance of the ball on a flat board. Each agent controls the velocity and position of the ball through lifting up or putting down one of four edges of the board. Such actions are achieved through a communication based on TCP/IP protocol between agents PCs and the graphics server, as shown in Fig.1.

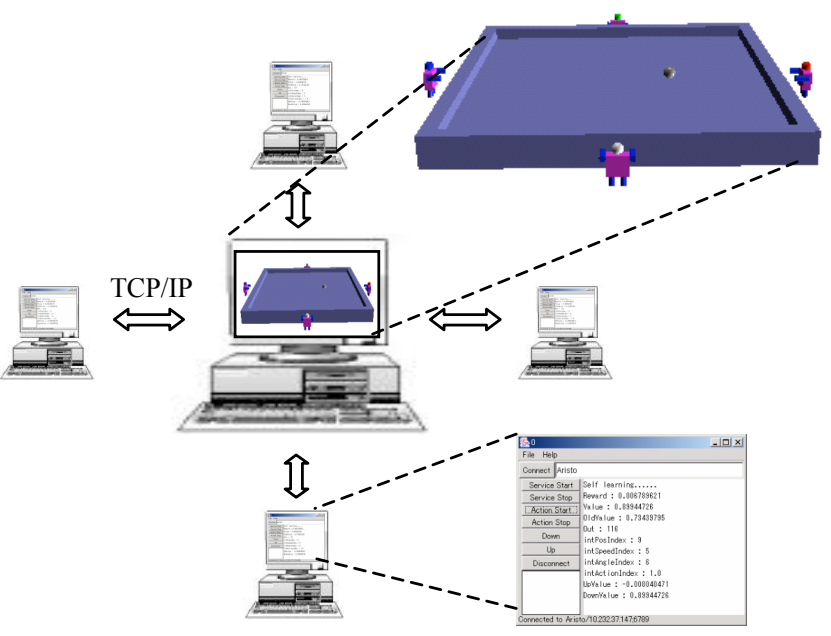

Fig. 1 Testbed for social reinforcement learning. Four agents communicate with the sever through network.

To perform this task, we assume our software agents are intelligent entities with individual reinforcement learning capabilities and social learning capabilities. Through numerous individual reinforcement trainings and social reinforcement learning, they will know how to control the panel to avoid the ball rolling out of the board. In our experiment testbed, environment variables such as position, velocity of the ball and declined angles of the board are shared among all agents: such environment variables as the current position and velocity of the ball 
and angle of the board. Some other variable such as the friction between the ball and the board are unknown to all agents.

The agents' environment is dynamic, and some environment variables and participants may vary from time to time (i.e., fluid participation). For instance, the friction between the ball and the board can be changed without agents notice. Furthermore, the member of the agents participating in the collaboration is not fixed at all, but a newcomer agent may enter into the team, or some agents who has acquired the control know-how may withdraw from the team abruptly without informing others. To ensure co-learning among agents converge eventually, a rapidly converging algorithm is required so that agent's learning can catch up with the dynamical changes of environment variables within a reasonable time span.

The results of this experiment showed that three kinds of social reinforcement learning approaches - individual reinforcement, observing reinforcement and vicarious reinforcement - are worthwhile in exploring in a multi-agent domain. We studied the performance of social reinforcement learning approaches with respect to their learning efficiency of accelerating converging speed in multiagent environment.

\subsection{Gaps between Designer's and User's Views}

In order to avoid the mismatch between a designer's view and a user's view, Degani and Heymann has proposed a composite model analysis to detect such a contradiction between a given machine model and an expected user model based on the graph theoretical manipulation of those diagrams [6]. They showed two kinds of states to be detected in the composite model that is formed from a given machine model and a user model in order to avoid the misconception of a user in using a machine; error states and blocking states. An error state is a state that misguides a user's recognition as normal although an actual state is anomalous, and vice versa. A blocking state is a state in which a user cannot notice some state transitions may possible under this state, but actually a machine is able to change its states automatically wherein. In this case, a user may feel confused very much wondering why automation is behaving like this. This is a typical situation caused by a well-known "automationinduced surprise" and the interface should be designed so that a user should not face such a surprise.

Using this technique, we represented the complex control logics of ACC of the commercial automotive as shown in Fig.2. Wherein, the states with bold characters represents different mode under which a user driver is forced to drive. Italics shown in the transitions represent conditions when the transition among the mode occurs. Depending upon the mode an action taken by a driver will have a different reaction of the automotive. Therefore, if a driver grasps a current mode wrongly and takes some actions, the resulted behaviors of the automotive will differ from what he/she expected. With respect to the safety problem of such an advanced automated automotive, we are approaching two kind of problems; one is to improve the misconception of the human driver's mode recognition errors, and the other one is to make a pre-analysis on investigating into what's type of novel misconception may be possibly caused by the addition of more complicated automated functions.

As for the former problem we installed the same logics with the ACC into our driving simulator and made a series of experiments using human subjects. From the experiments, it is shown that some modes formed by ACC are apt to be misclassified by the human subjects, although the mode changes are explicitly displayed in the interface to the drivers. Drivers are mainly engaged in their driving tasks paying little attention to the status of ACC. They can know the status of ACC from the interactions with the automotive equipments (i.e., steering, breaking, accelerating pedal, etc.) and by noticing their reactions to a driver's exerting driving operation. We develop a new method for notifying the change of modes noticing the driver's sensitivity to such interactive reactions, and verified that that can improve the rates of correct recognition of mode changes of the ACC.

As for the second problem, we dealt with a function of accepting a driver's changing the set speed while the ACC with the previous setting is working (this is sometimes called a "reaccelerating" function). Such a complex control logics may induce the human driver to reason on predicting behaviors using a wrong user model that is contradictory with an actual behavior determined by a machine model. For this problem, we applied the composite model analysis and develop a method for detecting such human-automation discrepancy at the design stage [7].

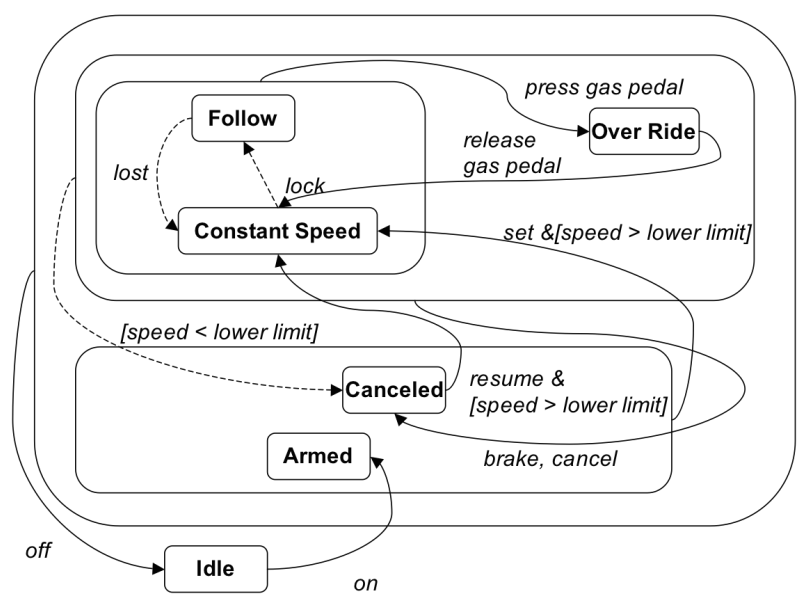

Fig.2 Complicated control logics of the ACC shown as Machine model.

\subsection{Knowledge Sharing within Work Community}

All types of technical know-how, which in essentials depend on one's personal experiences, are so difficult to be completely codified into and transferred through any explicit descriptions like documents. On the other hand, it is much required to share such individuals' expertise in the organizations efficiently so that companies can develop their own technical capabilities and maintain their 
competitiveness in the markets. In this context, a lot of efforts have ever been made to construct a database, which stores certain data related to the practical work skills. But those platforms are sustainable only when they can provide users with some effective accesses to what they are most likely to want. That is to say, the harder the users have to be occupied with their search and retrieval, the more they would be discouraged to use such systems. We approached the above issue in designing a knowledge management platform for sharing technical know-how in specific organizations. The point is how to leverage the information processing technologies for developing a kind of facilitating system that can mediate different user experiences. As a testbed for this approach we adopted know-how on the tuning of the automated inspection systems fostered within the community of the suppliers of the inspection systems.

An automated visual inspection system discriminates defective items on production lines by applying several installed inspection algorithms to measurements of its vision sensors. The principle of the system is shown in Fig.3. When newly programming or tuning these algorithms for proper inspections, a system engineer has to exploit a variety of technical know-how, such as how to select and combine diagnostic criteria suitable to catch enough common features of defective/non-defective parts in response to the sensing conditions and/or the characteristics of the inspected products. Therefore, performance of those programs is very dependent on and enclosed in each engineer's experiences. In order to share and reuse such personal expertise among their community, their work practices should be recorded in some way and referred to again easily by other community members.

For enabling the sharing of those personal experiences, supplier companies are beginning to construct a database to store the work reports on programming inspection systems. In addition to descriptions on a responsible worker, inspection machines servicing, and so on, every account record in the database contains explanations on an attacked problem, and ways (or tricks) of coping with it, both of which are supported by some selected picture images for inspection processing. However, there are difficulties in making use of such records as clues to solve a problem that stares an engineer in the face. Those who are asking some clues from the database must not basically know any solutions to the problems confronting them. Hence, they have to depend on some "surface words" representing inspected objects, when they make search queries. But in such situations, simple keyword matching would give no helpful information because their community is so confined in a specific domain that other engineers are more likely to have never experienced the identical problems with theirs. That will upset those users in determining keywords composing their search queries.

From this perspective, rather than words themselves, latent semantic connections among them should be considered when searching the database, and it will be a good support for such users. At the same time, the users' burden should also be taken account of when finding out what meets their needs from many alternatives in search results. On the basis of these motivations, we are approaching a new concept of the knowledge management framework for sharing technical know-how in organizations as shown in Fig.4 [8].

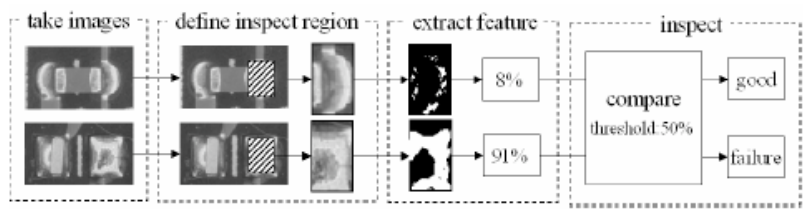

Fig.3 Visual inspection system for the printed circuit board.

Our proposing framework is composed of two different semantic networks with spreading activation mechanisms: the Query Extension Network (QE Net) and the Query Suggestion Network (QS Net). Each of them represents a different aspect of relevance among keywords that can be used in database search queries. The QE Net is intended to extend a search by a user's query through its periphery based upon the associative relations of words in the domain ontology. The QS Net is intended to present a user with other keywords for his next search query, which might help his exploration advance to what he wants. The interaction of both functions through search activities by an individual user is expected to provide effective navigations for his explorations in the forest of expertise intertwined.

\subsection{Ecological Interface Design}

The recent serious industry accidents have revealed the importance of the higher level of the cognitive competence of actors in order to keep the highest degree of safety of complex system such as nuclear power plants, nuclear fuel processing plants, chemical process plants and airplanes. And importance has been recognized of supporting actors' deep understanding of the functional structure and dynamics of the system and ad hoc planning based on it. This is true not only for those safety-critical technological systems, but also for other manufacturing control devices. Those may not be safety-critical, but the easiness of the operation to usual workers (i.e., even to less-skilled workers) will be of fundamental importance considering the scarceness of the experienced human resources. The ecological interface design concept is a promising idea for this purpose. The objective of ecological interface design is to support adaptation of the actors to work situations and conditions that cannot be anticipated at the design stage. The objectives is intended to be achieved through making visible the internal ecology of work and, to be effective, allows direct perception of the state of the world in the light of the current goals, as well as the boundaries of the acceptable performance.

We have studied on improving interface designs to facilitate unskilled workers' awareness on the latent but essential structures composing the controlled system's behaviors. The testbed control device used here is the Computer-Controlled Checkweighing (CCW) systems. As those systems require adequate parameters to be tuned for 


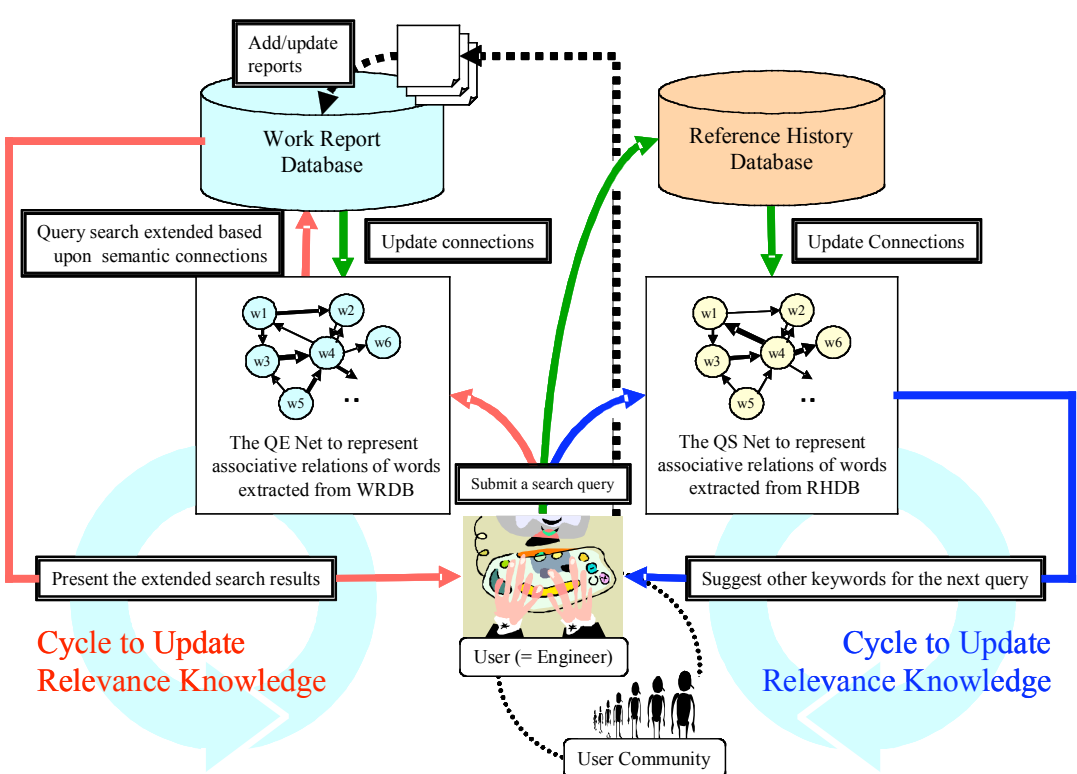

Fig.4 Knowledge management system for visual inspection system of OMRON Co.

their effective operations, there have been large performance gaps between experts and average workers. Dealing with this problem, the analysis using the Rasmussen's decision ladder model was conducted from questionnaires and interviews to sort out the skill factors between them, and to improve the user interface design. The results confirmed that the experts' deeper knowledge and insights on the states of the weigher systems in relation to their available operations. Further, we investigated into the correlation between the skill performance and the workload incurred to the operator. The latter was measured using a technique of NASA-TLX (Task Load Index). The results revealed very clear positive correlation between them [9].

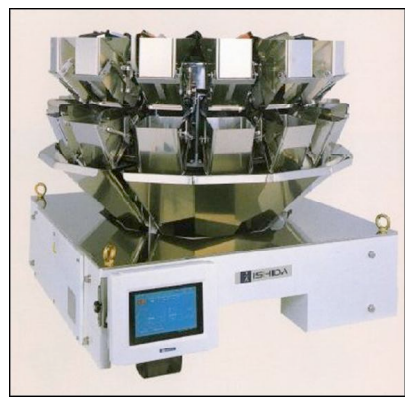

Fig.6 Computer-Controlled Checkweighing (CCW) system of Ishida Co..

Based upon those results, we designed an ecological interface system for the $\mathrm{CCW}$. The idea wherein is to externalize the invisible cues that the experts grasp during their skillful operations on the display. Such invisible cues were identified through functional analysis of the weighing process of the device. Fig. 6 shows the overview of the CCW, and the invented interface is now patent pending. By visualizing what to be sought and by affording what operations to be taken to the operator with direct perception,
Fig.5 Computer-Controlled Checkweighing this revised interface contributes much to the improvement of the operations, which was confirmed in the experiments. In this way, our proposed interface functions well for linking flexible human judgments and automated capabilities that the $\mathrm{CCW}$ has originally has. That is, this is establishing real human-automation collaboration.

\section{CONCLUSIONS}

The common perspective towards realizing the human-automation collaboration is how to design a human-in-the-loop-system, rather than a human-out-of- the-loop system. In order to establish ideal human-automation collaboration, we have to resolve two fundamental technical issues: one is to let the machine adaptable to the variability of the interactions with the human, and the other one is also to let the human evolvable by accumulating their experiences. The former is realized by developing social learning algorithms, while the latter is attained by making visible the invisible in the interface systems.

\section{REFERENCES}

[1] Billings, C.E. (1997). Aviation Automation: The Search for a Human Centered Approach, Lawrence Erlbaum Ass.

[2] Sheridan, T.B. (1997). Human-Centered Automation: Oxymoron or Common Sense?, Proc, of IEEE Int. Conf. on System, Man and Cybernetics, Vancouver, Canada.

[3] Conte, R.: Intelligent Social Learning. Journal of Artificial Societies and Social Simulation, 4(1).

[4] Sutton, R.S. and Barto, A.G. (1998). Reinforcement Learning: An Introduction. MIT Press, Cambridge, MA.

[5] Wei, Q. and Sawaragi, T.:(2004). A Social View to Multiagent Reinforcement Learning, Transactions of SICE, 40(3).

[6] Heymann, M. and Degani, A. (2002). On the construction of human-automation interfaces by formal abstraction, Symposium on Abstraction, Reformulation and Approximation, Kananaskis Mountain Lodge, Kananaskis, Alberta, Canada.

[7] Sawaragi, T. Horiguchi, Y. and Hina, A. (2005). Analytical Approach to Correct and Succinct Interface Design for Safety-Monitoring in Cockpit, Proc. of the HCI International 2005 Conference, CD-ROM.

[8] Horiguchi, Y. Sawaragi, T., Kaneda, Y. and Nakajima, A. (2004). Knowledge Management Framework to Share Technical Know-How in Organization, Proceedings of 2004 IEEE International Conference on Systems, Man \& Cybernetics, pp.1436-1441.

[9] Horiguchi, Y. Sawaragi, T. et al. (2006). Development of Smart User Interface for Operating Multithread Weighers: Skill Analysis through Human Model of Information Processing Activities, Journal of Human Interface, Transactions of Human Interface Society (in submission). 